\title{
Fine wakefield structure in the blowout regime of plasma wakefield accelerators
}

\author{
K. V. Lotov \\ Budker Institute of Nuclear Physics, 630090, Novosibirsk, Russia
}

(Received 19 December 2002; published 6 June 2003)

\begin{abstract}
For simulations of plasma wakefield acceleration (PWFA) and similar problems, we developed twodimensional fully electromagnetic fully relativistic hybrid code LCODE. The code is very fast due to explicit use of several simplifying assumptions (quasistatic approximation, ultrarelativistic beam, and the symmetry). With LCODE, we make high-resolution simulations of the blowout regime of PWFA and study the temperature effect on the amplitude of the accelerating field spike.
\end{abstract}

DOI: 10.1103/PhysRevSTAB.6.061301

\section{INTRODUCTION}

Acceleration and focusing of particles with plasmas is of a great interest because the field strength in the plasma can be made several orders of magnitude higher than that in conventional accelerating structures or focusing lenses [1-4]. The accelerating field in the plasma can be excited either by a powerful laser pulse (or several pulses) [5-7] or by a charged particle beam (usually electron beam) [8]. The latter variant is usually termed PWFA (plasma wakefield accelerator). Here we consider the wave excitation by electron beams.

To compete with conventional accelerators, the plasma accelerator must have high efficiency, high final energy, and a good quality of the accelerated beam. Various configurations of the drive beam were proposed in order to meet these requirements [8-12]. One option is to use a single high-density drive bunch (so-called blowout regime) [12]. In this regime, the head of the driver ejects all of the plasma electrons from its propagation channel, and the most part of the beam propagates in the electronfree region. The blowout regime was extensively studied both theoretically [12-18] and experimentally [4,19-24]. It has a number of good features. The linear focusing force is good for preservation of the accelerated beam emittance. The accelerating field, which does not depend on radius inside the ion channel, helps to minimize the energy spread. The maximum value of the accelerating field is much greater than the decelerating field within the driver, which gives hope to get an energy gain far in excess of the driver particle energy.

In this paper we study the blowout regime of PWFA. With the two-dimensional code LCODE [25], we calculate the wakefield structure near the point of the maximum accelerating field and separate numerical and physical effects. We show that the enormously high spike of the accelerating field is an artifact of adopted models and cannot be used for acceleration of a reasonably large number of particles.

The paper is organized as follows. In Sec. II we describe the code and compare it with other codes $[15,17,18]$ by simulating the SLAC E-157 experiment $[4,23]$. In Sec. III we present high-resolution simulations of the first
PACS numbers: $52.40 . \mathrm{Mj}, 52.35 . \mathrm{Mw}, 52.65 . \mathrm{Ww}$

wakefield period and discuss the temperature effect on the amplitude of the accelerating field spike.

\section{THE CODE}

Two-dimensional fully electromagnetic fully relativistic code LCODE [25] supports both Cartesian and cylindrical geometries. For definiteness, we write out all equations for the cylindrical case.

We use the cylindric coordinates $(r, \varphi, z)$ and the comoving simulation window (Fig. 1). Since the length scale of beam evolution is typically much longer than the bunch length, we work in the so-called quasistatic approximation [26,27]. Namely, when calculating the plasma response we consider the beam as "rigid" and find the fields as functions of $r$ and $\xi=z-c t$, where $c$ is the speed of light. Then we use these fields to modify the beam, etc.

The beam is modeled by macroparticles. The plasma can be modeled either as a relativistic electron fluid or by macroparticles. The beam model and electron fluid model are described in detail in [27]. Briefly, each beam macroparticle is characterized by $r$ and $\xi$ coordinates $\left(r_{b}\right.$ and $\left.\xi_{b}\right), r$ and $z$ momenta $\left(p_{b r}\right.$ and $\left.p_{b z}\right)$, and angular momentum $\left(M_{b}\right)$. They are changed every time step:

$$
\begin{gathered}
\frac{d r_{b}}{d t}=v_{b r}, \quad \frac{d \xi_{b}}{d t}=v_{b z}-c, \quad \frac{d M_{b}}{d t}=0, \\
\frac{d p_{b r}}{d t}=q_{b}\left(E_{r}-B_{\varphi}\right), \quad \frac{d p_{b z}}{d t}=q_{b} E_{z}, \\
\vec{v}_{b}=\frac{c \vec{p}_{b}}{\sqrt{m_{b}^{2} c^{2}+p_{b r}^{2}+p_{b z}^{2}+M_{b}^{2} / r_{b}^{2}}},
\end{gathered}
$$

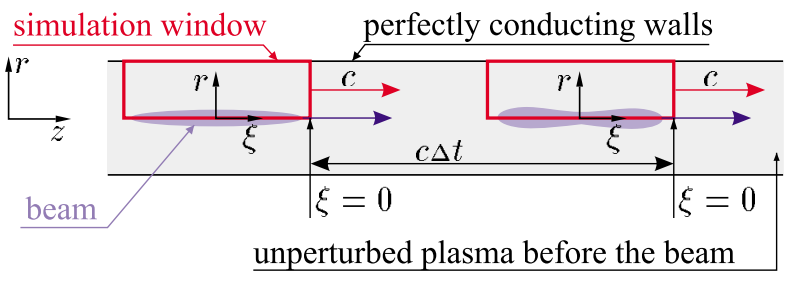

FIG. 1. (Color) Geometry of the problem. 
where charges $q_{b}$ and masses $m_{b}$ are equal for all macroparticles. The time step $\Delta t$ for beam particles can be made large enough for highly relativistic beams (Fig. 1) to speed up simulations.

The macroparticle model of the plasma was specially developed for simulations of the nonlinear PWFA and similar problems. This is a Lagrangian model rather than a conventional particle-in-cell model. Namely, each plasma macroparticle is characterized by six quantities: transverse coordinate $(r)$, three components of the momentum $\left(p_{r}, p_{\varphi}\right.$, and $\left.p_{z}\right)$, mass $M$, and charge $q$. The longitudinal coordinate $(\xi)$ is not a parameter, but an argument. Thus, a plasma macroparticle presents a "particle tube," i.e., a group of real particles started from a given radius (Fig. 2). Since, in the quasistatic approximation, the beam is unchanged while we calculate the plasma response, all particles started from $r_{0}$ copy the motion of each other, and their parameters $\left(r, p_{r}, p_{\varphi}\right.$, and $p_{z}$ ) can be found as functions of $\xi$.

Parameters of plasma macroparticles are initialized ahead of the beam (at $\xi=0$ ) and then calculated slice by slice:

$$
\begin{aligned}
& \frac{d \vec{p}}{d \xi}=\frac{d \vec{p}}{d t} \frac{d t}{d \xi}=\frac{q}{v_{z}-c}\left(\vec{E}+\frac{1}{c}[\vec{v} \times \vec{B}]\right), \\
& \frac{d r}{d \xi}=\frac{v_{r}}{v_{z}-c}, \quad \vec{v}=\frac{\vec{p}}{\sqrt{M^{2}+p^{2} / c^{2}}} .
\end{aligned}
$$

Plasma current and charge density are obtained by summation over plasma macroparticles lying within a given radial interval:

$$
\vec{j}=A \sum_{i} \frac{q_{i} \vec{v}_{i}}{c-v_{z, i}}, \quad \rho=A \sum_{i} \frac{q_{i}}{c-v_{z, i}},
$$

where $A$ is a normalization factor. The denominator in (6) appears since the contribution of a particle tube to the density and current depends on the macroparticle speed in the simulation window.

Knowing $\vec{j}$ and $\rho$ at some $\xi$, we obtain the fields in this layer from Maxwell equations which, under the assumption

$$
\partial / \partial \xi=\partial / \partial z=-\partial / \partial(c t)
$$

take the form

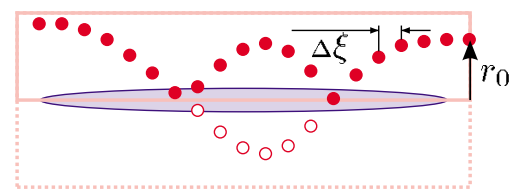

FIG. 2. (Color) Trajectory of a plasma macroparticle in the simulation window.

$$
\begin{gathered}
\frac{1}{r} \frac{\partial}{\partial r} r E_{r}=4 \pi\left(\rho+\rho_{b}\right)-\frac{\partial E_{z}}{\partial \xi} \\
\frac{1}{r} \frac{\partial}{\partial r} r B_{r}=-\frac{\partial B_{z}}{\partial \xi} \\
\frac{\partial\left(E_{r}-B_{\varphi}\right)}{\partial \xi}=\frac{\partial E_{z}}{\partial r}=\frac{4 \pi}{c} j_{r} \\
\frac{\partial B_{z}}{\partial r}=-\frac{4 \pi}{c} j_{\varphi}, \quad E_{\varphi}=-B_{r} .
\end{gathered}
$$

Here $\rho_{b}$ is the beam charge density, and we neglect the components $j_{b r}$ and $j_{b \varphi}$ of the beam current.

To provide stability of the algorithm, we solve in finite differences, instead of (8) and (9), the following equations:

$$
\frac{\partial}{\partial r} \frac{1}{r} \frac{\partial}{\partial r} r E_{r}-\frac{\omega_{p}^{2}}{c^{2}} E_{r}=4 \pi\left(\frac{\partial\left(\rho+\rho_{b}\right)}{\partial r}-\frac{1}{c} \frac{\partial j_{r}}{\partial \xi}\right)-\frac{\omega_{p}^{2}}{c^{2}} \tilde{E}_{r},
$$

$$
\frac{\partial}{\partial r} \frac{1}{r} \frac{\partial}{\partial r} r B_{r}-\frac{\omega_{p}^{2}}{c^{2}} B_{r}=\frac{4 \pi}{c} \frac{\partial j_{\varphi}}{\partial \xi}-\frac{\omega_{p}^{2}}{c^{2}} \tilde{B}_{r},
$$

where $\tilde{E}_{r}$ and $\tilde{B}_{r}$ are the fields at the previous layer, and $\omega_{p}=\sqrt{4 \pi n_{0} e^{2} / m}$ is the unperturbed plasma frequency. These equations are obtained by differentiation of (8) and (9), and substitution of (10) and (11) into the result. Addition of the fields (with or without the tildes) to both sides of the equalities does not change the equations and has the effect only when we proceed to final differences.

The boundary conditions for Eqs. (10)-(13) are that of a perfectly conducting tube of the radius $r_{\max }$ :

$$
\begin{array}{cc}
E_{r}(0)=B_{r}(0)=B_{\varphi}(0)=0, & E_{z}\left(r_{\max }\right)=0, \\
\int_{0}^{r_{\max }} 2 \pi r B_{z} d r=\pi r_{\max }^{2} B_{0}, & B_{r}\left(r_{\max }\right)=0,
\end{array}
$$

where $B_{0}$ is an external longitudinal magnetic field, if any (the presence of this field does not change the symmetry of the system).

The plasma response is calculated layer by layer towards the decreasing $\xi$ [from right to left in (Fig. 1)]. We use (4) -(6) to predict $\vec{j}$ and $\rho$ at the next layer, calculate the fields at this layer, move plasma macroparticles using the average fields, then correct $\vec{j}, \rho$, and fields at the new layer. The algorithm allows easy shortening of the $\xi$ step in the regions of a fine field structure. The shortening is made automatically when the current density $\left|j_{z}\right|$ exceeds some threshold value. The shift of beam particles according to Eqs. (1)-(3) is made in parallel with the calculation of the plasma response, so that every beam time step 


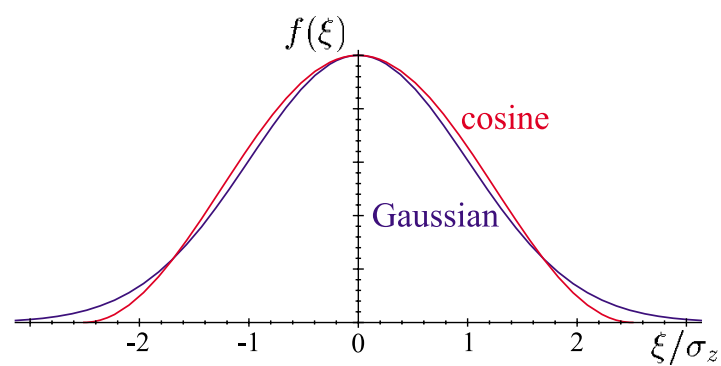

FIG. 3. (Color) Cosine and Gaussian distributions.

requires only one computing passage of the simulation window.

The code allows an arbitrary initial distribution of beam particles over the simulation window, but we usually take the initial beam density of the form

$$
n_{b}=\frac{n_{b 0}}{2} e^{-r^{2} / 2 \sigma_{r}^{2}}\left[1-\cos \left(\frac{2 \pi \xi}{L}\right)\right], \quad-L<\xi<0 .
$$

The cosine distribution over $\xi$ is more convenient than the Gaussian one because it smoothly vanishes outside an interval of a finite length. For

$$
L=2 \sqrt{2 \pi} \sigma_{z}
$$

the distribution (16) is close to the Gaussian one

$$
n_{b}=n_{b 0} \exp \left(-\frac{r^{2}}{2 \sigma_{r}^{2}}-\frac{(\xi+L / 2)^{2}}{2 \sigma_{z}^{2}}\right)
$$

(Fig. 3) and contains the same number of particles.

Because of the explicit use of the simplifying assumptions (quasistatic approximation, ultrarelativistic beam, and the symmetry), the code is very fast. To demonstrate this, we simulate the SLAC E-157 experiment [4,23]. We take the beam and plasma parameters close to those used in earlier simulations $[15,17,18]$ (Table I). To simulate the accelerated particles correctly, we take the half-Gaussian trailing edge of the beam, while the front edge is the halfcosine. Similarly to $[15,17]$, we use a square grid of the size $0.05 c / \omega_{p}$, nine macroparticles per cell for the plasma, and 25 macroparticles for the beam (near the axis). The time step for the beam corresponds to $1.4 \mathrm{~cm}$.

The simulation results are shown in Fig. 4. They are in good agreement with [15,17]. Even the narrow field spike

TABLE I. Beam and plasma parameters.

\begin{tabular}{lc}
\hline \hline Number of beam particles, $N$ & $3.7 \times 10^{10}$ \\
Beam radius, $\sigma_{r}$ & $70 \mu \mathrm{m}$ \\
Beam half-length, $\sigma_{z}$ & $0.63 \mathrm{~mm}$ \\
Beam energy, $W_{b}$ & $30 \mathrm{GeV}$ \\
Plasma density, $n_{0}$ & $2.1 \times 10^{14} \mathrm{~cm}^{-3}$ \\
Plasma length, $L$ & $1.4 \mathrm{~m}$ \\
\hline \hline
\end{tabular}
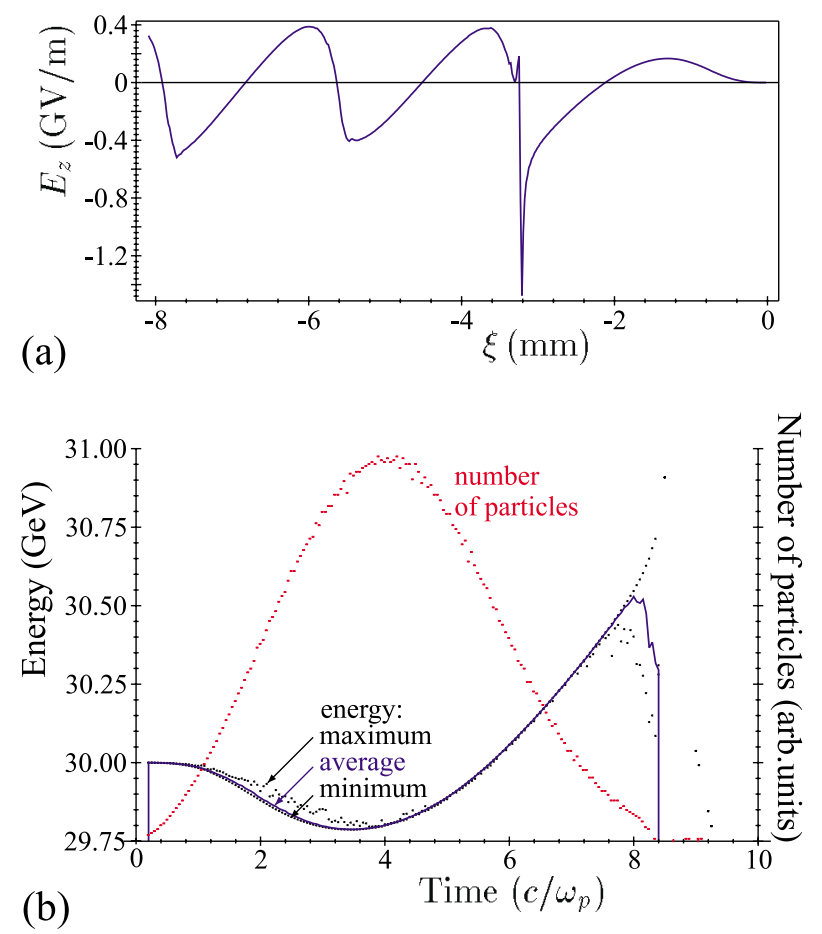

FIG. 4. (Color) (a) Longitudinal electric field, (b) energy and number of beam particles after $1.4 \mathrm{~m}$ plasma for the lowresolution simulation of the E-157 experiment.

just behind the maximum accelerating field observed in PEGASUS and OSIRIS simulations [15] is reproduced. The only drastic difference is that the full beam dynamics run [Fig. 4(b)] takes about 5 min on a desktop Pentium-II rather than days on a multiprocessor Cray [15].

\section{FINE STRUCTURE OF THE WAKEFIELD}

Simulations of the blowout PWFA with various codes are in perfect agreement everywhere except the region of the sharp accelerating field spike just behind the beam. The dependence of the spike amplitude on the grid size [15] and a noisy electric field after the spike suggest that the resolution of earlier runs is not sufficient for correct description of this region. At the same time, it is this spike that promised ultrahigh accelerating gradients and greatly contributed to attractiveness of the blowout regime. With this motivation, we make high-resolution simulations of the near-spike region.

We take the same beam parameters (Table I), but a much denser grid. The simulation window, in units of $c / \omega_{p}$, is 20 (in $\xi$ ) $\times 5$ (in $r$ ). Both $r$ and $\xi$ steps are $0.0025 c / \omega_{p}$, and near the spike the $\xi$ step is automatically decreased down to $2.5 \times 10^{-5} c / \omega_{p}$. The beam is modeled by $3.4 \times 10^{5}$ macroparticles, and $4 \times 10^{4}$ plasma macroparticles are used (20 macroparticles per cell).

The simulation results are shown in Fig. 5. The graphs for zero plasma temperature $\left(T_{e}=0\right)$ are shown in the left 


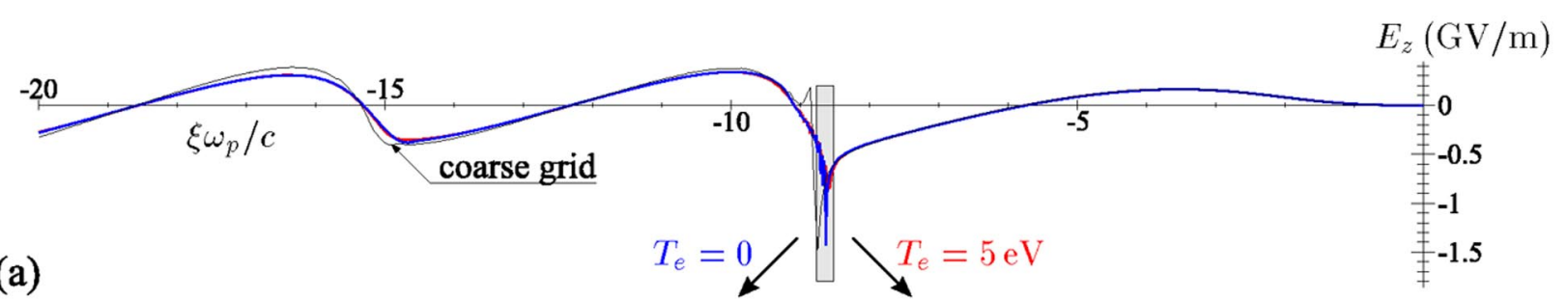

(b)

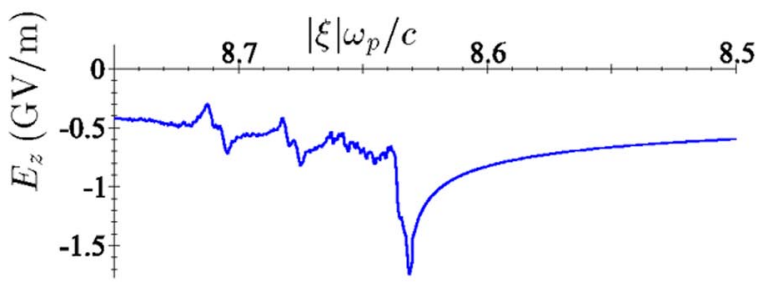

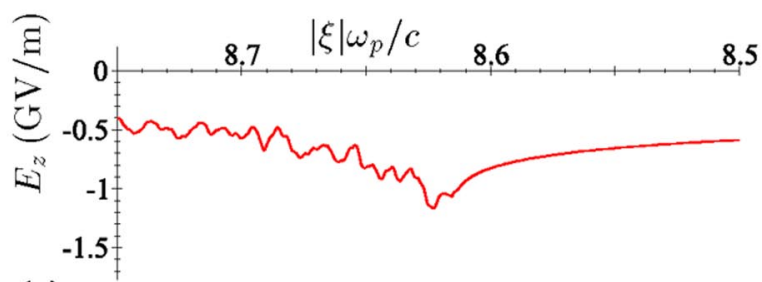

(c) (d)

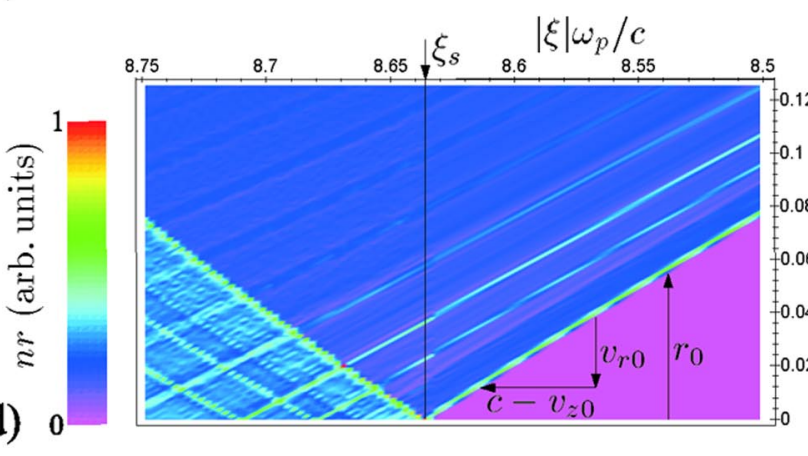

(f)

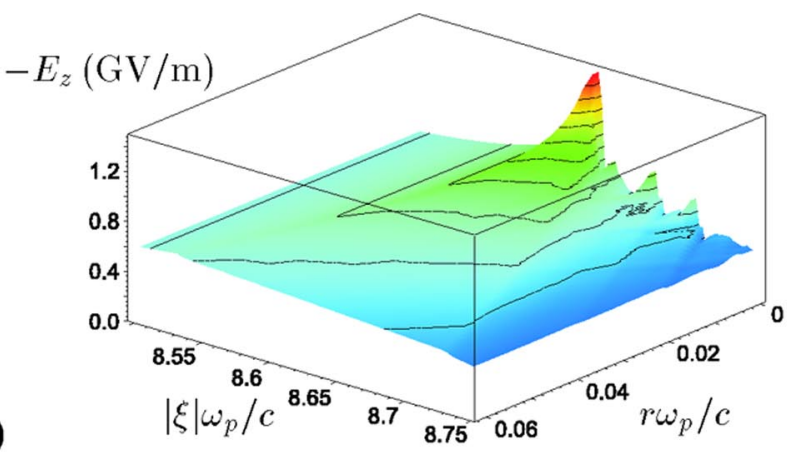

(e)

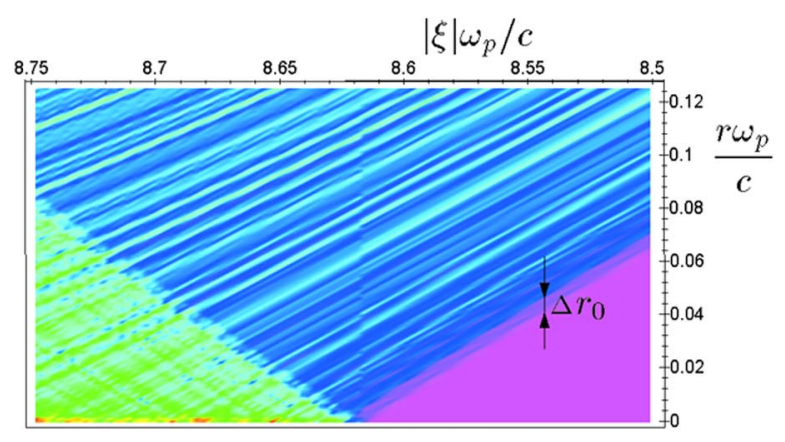

(g)

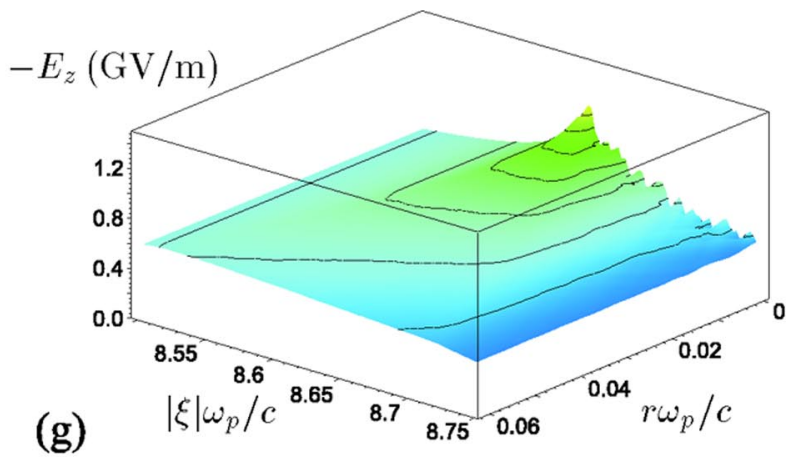

$\left(E_{r}-B_{\varphi}\right) / \sqrt{4 \pi n_{0} m c^{2}}$

(h)

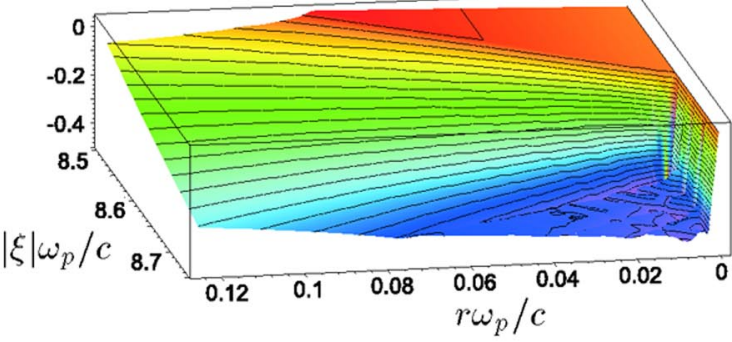

$\left(E_{r}-B_{\varphi}\right) / \sqrt{4 \pi n_{0} m c^{2}}$

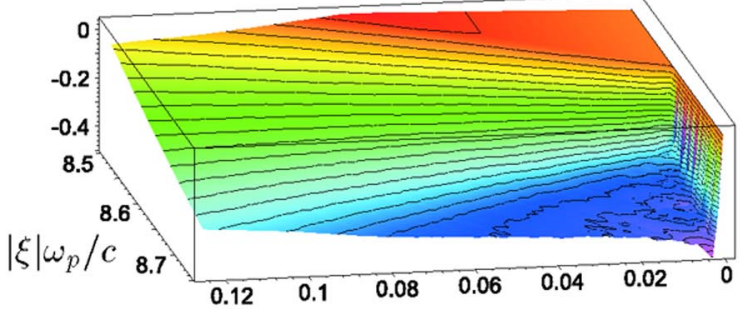

(i)

FIG. 5. (Color) Plasma and field behavior in the vicinity of the highest accelerating field: (a) on-axis values of $E_{z}$ for different runs and the location of the magnification window; (b),(c) on-axis values of $E_{z}$ in the region of the field spike; (d),(e) plasma electron density (multiplied by $r$ for better visibility); (f),(g) longitudinal electric field; and (h),(i) focusing force with zero (b),(d),(f),(h) and nonzero (c),(e),(g),(i) plasma temperatures. 
column; the right column shows $T_{e}=0.1 \mathrm{eV}$ according to E-157 data published in [23]. The location of the detailed window is indicated in Fig. 5(a). The wakefield calculated in Sec. II with a coarse grid is also shown in Fig. 5(a) for comparison.

It is seen from Fig. 5(a) that the initial plasma temperature has a visible effect on the wakefield only in the region of the field spike. The spike is suppressed by the temperature [Figs. 5(b) and 5(c)]. The reason why this happens is explained by maps of the plasma electron density [Figs. 5(d) and 5(e)], where the product $n r$ is shown; the factor of $r$ compensates the geometry-induced singularity of the density. It is seen that the field growth is stopped when some plasma electrons arrive at the axis. For the initially cold plasma, the boundary of the blowout region is sharp. This boundary, when approaching the axis, produces a high current density

$$
j_{r}=-e n v_{r} \propto 1 / r
$$

(because of the singular density growth at a nearly constant radial speed) and, according to (10), the singularity of $E_{z}$. For the warm plasma, the edge of the blowout region is diffuse, currents of inward and outward moving electrons almost cancel each other, and no field singularity appears.

In the transverse direction, the main field spike is also very narrow [Figs. 5(f) and 5(g), notice the scale]. In the regions of a nonzero plasma electron density, a strong defocusing force acts on the beam [Figs. 5(h) and 5(i)]. As a result, the region favorable for particle acceleration is very narrow in the vicinity of the spike (the triangular area of positive values in Figs. 5(h) and 5(i)], and we have a trade-off between the width of the favorable cross section and the accelerating field in it. Note that behind the spike the negative focusing force is almost constant up to very small radii, which means huge defocusing gradients near the axis.

The above picture of spike formation suggests a simple analytical dependence of the spike amplitude on the plasma temperature. Assuming the scaling (19) is true, we can find, for zero plasma temperature, the on-axis field at the leading edge of the spike:

$$
\begin{aligned}
E_{z} & =-\frac{4 \pi}{c} \int_{0}^{\infty} j_{r} d r \approx \frac{4 \pi e}{c} \int_{r_{0}}^{r_{\infty}} \frac{n_{\infty} v_{r} r_{\infty}}{r} d r \\
& \approx A_{1}+A_{2} \ln r_{0}, \\
A_{2} & >0
\end{aligned}
$$

where $r_{0}$ is the radius of the electron-free region [Fig. 5(d)], $r_{\infty}$ is a typical radius of the perturbed plasma, $n_{\infty}$ is the electron density there, and $A_{i}$ are some constants. For straight electron trajectories [which is true in our case, as follows from Fig. 5(d)], we have

$$
r_{0}(\xi)=\frac{v_{r 0}}{c-v_{z 0}}\left(\xi_{s}-\xi\right)
$$

where $v_{r 0}<0$ and $v_{z 0}$ are electron velocities near the spike, and $\xi_{s}$ is the coordinate of the field extremum. Whence

$$
E_{z} \approx A_{3}+A_{2} \ln \left(\xi-\xi_{s}\right),
$$

and we observe a logarithmic singularity of the longitudinal electric field.

When the plasma has a nonzero initial temperature, electrons slightly deflect from their zero-temperature trajectories, and some of them arrive at the axis earlier than in the zero-temperature case. Typical deviation of an electron at the distance $\left|\xi_{s}\right|$ from the beam head is

$$
\Delta r_{0} \sim \frac{\left|\xi_{s}\right|}{c} \sqrt{\frac{T_{e}}{m}}
$$

[Fig. 5(e)]. At $r_{0} \sim \Delta r_{0}$, the logarithmic increase of $\left|E_{z}\right|$ stops, and we observe the maximum field

$$
E_{z, \max } \approx A_{1}+A_{2} \ln \Delta r_{0} \approx A_{4}+A_{5} \ln T_{e}, \quad A_{5}>0 .
$$

Coefficients $A_{i}$ here depend on the driver shape, current, and radius.

This nonrigorously obtained scaling is surprisingly well confirmed by simulations (Fig. 6). The thin line in Fig. 6 is determined by the formula

$$
E_{z, \max }(\mathrm{GV} / \mathrm{m})=-0.98+0.14 \log T_{e}(\mathrm{eV}) .
$$

At high plasma temperatures, we observe a systematic deviation of the field amplitude from this scaling; since the ratio $r_{\infty} / \Delta r_{0}$ is not very high there, wide plasma regions contribute to the integrals (20), and the logarithmic precision of our estimates becomes worse. At low temperatures, $\Delta r_{0}$ approaches the grid step, and numerical noises come into play.

As follows from the above, the main field spike can be used for acceleration only in idealized conditions of a perfectly symmetric and cold plasma and a very narrow beam. In real experiments the conditions are rather far from the ideal ones. The plasma could have transverse density gradients, and the beam is not perfectly cylindrical and straight. These effects cannot be analyzed with a two-dimensional code, but we can expect that account of

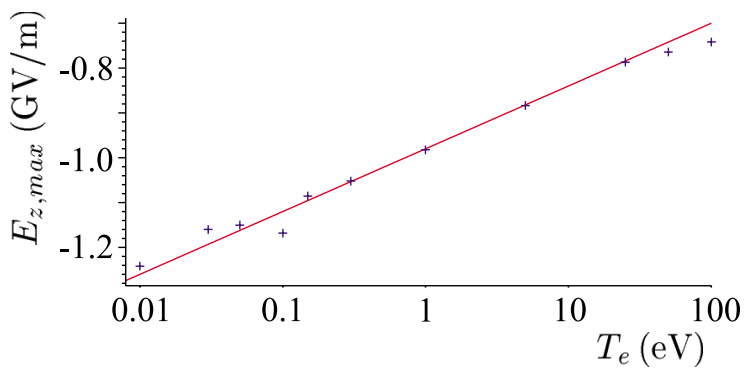

FIG. 6. (Color) The temperature dependence of the spike amplitude: simulation (crosses) and approximation (line). 
three-dimensional effects will make the situation worse, no additional length scale or field scale will appear, and the maximum accelerating field behind the beam will not be an order of magnitude higher than the decelerating field within the beam.

\section{ACKNOWLEDGMENTS}

This work was supported by a Science support foundation grant, an SB RAS grant for young researchers, and the Russian Foundation for Basic Research, Grants No. 00-15-96815 and No. 03-02-16160a.

[1] E. Esarey, P. Sprangle, J. Krall, and A. Ting, IEEE Trans. Plasma Sci. 24, 252 (1996).

[2] W. P. Leemans et al., Phys. Plasmas 5, 1615 (1998).

[3] D. Umstadter, Phys. Plasmas 8, 1774 (2001).

[4] C. Joshi et al., Phys. Plasmas 9, 1845 (2002).

[5] T. Tajima and J. M. Dawson, Phys. Rev. Lett. 43, 267 (1979).

[6] P. Sprangle, E. Esarey, J. Krall, and G. Joyce, Phys. Rev. Lett. 69, 2200 (1992).

[7] N. E. Andreev et al., JETP Lett. 55, 571 (1992).

[8] P. Chen, J. M. Dawson, R.W. Huff, and T. Katsouleas, Phys. Rev. Lett. 54, 693 (1985); 55, 1537(E) (1985).

[9] P. Chen et al., Phys. Rev. Lett. 56, 1252 (1986).
[10] R. D. Ruth, A.W. Chao, P. L. Morton, and P. B. Wilson, Part. Accel. 17, 171 (1985).

[11] K. V. Lotov, Nucl. Instrum. Methods Phys. Res., Sect. A 410, 461 (1998).

[12] J. B. Rosenzweig, B. Breizman, T. Katsouleas, and J. J. Su, Phys. Rev. A 44, 6189 (1991).

[13] N. Barov and J. B. Rosenzweig, Phys. Rev. E 49, 4407 (1994).

[14] J. Krall and G. Joyce, Phys. Plasmas 2, 1326 (1995).

[15] S. Lee, T. Katsouleas, R. Hemkel, and W. Mori, Phys. Rev. E 61, 7014 (2000).

[16] E. S. Dodd et al., Phys. Rev. Lett. 88, 125001 (2002).

[17] R. Hemkel, W. Mori, S. Lee, and T. Katsouleas, Phys. Rev. ST Accel. Beams 3, 061301 (2000).

[18] D. L. Bruhwiler et al., Phys. Rev. ST Accel. Beams 4, 101302 (2001).

[19] J. B. Rozenzweig et al., Phys. Rev. Lett. 61, 98 (1988).

[20] J. B. Rozenzweig et al., Phys. Rev. A 39, 1586 (1989).

[21] N. Barov, M. E. Conde, W. Gai, and J. B. Rosenzweig, Phys. Rev. Lett. 80, 81 (1998).

[22] N. Barov et al., Phys. Rev. ST Accel. Beams 3, 011301 (2000).

[23] M. J. Hogan et al., Phys. Plasmas 7, 2241 (2000).

[24] C. E. Clayton et al., Phys. Rev. Lett. 88, 154801 (2002).

[25] LCODE is available free at www.inp.nsk.su/ lotov/lcode.

[26] P. Sprangle, E. Esarey, and A. Ting, Phys. Rev. Lett. 64, 2011 (1990).

[27] K.V. Lotov, Phys. Plasmas 5, 785 (1998). 